\title{
Quantum Chemistry 'Without Orbitals' - An Old Idea and Recent Developments
}

\author{
Tomasz A. Wesolowski ${ }^{\star}$
}

\begin{abstract}
The role of one-electron functions known as orbitals in various theoretical methods used to describe molecules and complex materials at a quantum mechanical level is outlined in a historical perspective. A hierarchy consisting of three types of general formalism, ordered according to the importance of orbital-dependent expressions in the total energy, is presented. Two such formalisms, less known to the general chemistry community, are discussed in detail together with their recent applications in modelling complex systems: a) the orbital-free formulation of density functional theory, which does not use orbitals at all and which can be seen as the modern realization of the original ideas of Thomas and Fermi, and b) the density partitioning based formalism, in which the orbitals are used only for smaller parts of a larger system (subsystems). The emphasis is placed on the second type of formalism, a topic of strong interest of our Geneva group.
\end{abstract}

Keywords: Density functional theory $\cdot$ Kinetic energy functional $\cdot$ Orbital-free embedding $\cdot$ Orbitals

\section{Introduction}

Currently, computer modelling at a quantum mechanical level is used in almost all branches of chemistry. Experience shows that quantum chemistry and the concept of orbitals are inseparably linked [1][2]. As far as computer simulations are concerned, the terms quantum chemistry level and molecular orbital level are sometimes used as synonyms. This stems from two principal reasons: $i$ ) conceptual insight which the orbitals bring to various phenomena, $i i$ ) the fact that, with only a few exceptions, quantum mechanics based computational methods use explicitly molecular orbitals. The scope of this review is to outline recent progress towards development of such formalisms, which do not use orbitals to describe effects of quantum mechanical origin. The development of such formalisms is not only of academic interest. Modern chemistry deals frequently with large objects [3]. Molecules counting thousands of electrons can now be synthesized. The number of electrons in a typical object

${ }^{*}$ Correspondence: Dr. T.A. Wesolowski Department of Physical Chemistry

University of Geneva

30, Quai Ernest-Ansermet

$\mathrm{CH}-1211$ Genève 4

Tel.: +41227026957

Fax: +41227026518

E-Mail: tomasz.wesolowski@chiphy.unige.ch

http://lcta.unige.ch/ tomek/ of interest in biochemistry (large proteins or their aggregates) or nanotechnology reaches or even exceeds one million. Accurate computer modelling at a quantum mechanical level would enrich the set of research tools available for studying such systems. For very big systems, however, constructing and handling the wavefunction and/or orbitals implies formidable difficulties as far as practical computer modelling is concerned. The wavefunction is a rather complicated object. Managing it, assuring the orthogonality of the orbitals for instance, requires computational effort which scales more rapidly than the size of the system it describes. For systems comprising several thousands of atoms, these difficulties can be overcome to some extent owing to a rapid progress in the computer resources as well as in the development of faster computer implementations of conventional methods [4]. For large systems, a qualitatively new type of description of large systems, which would retain the capacity to describe effects of quantum mechanical origin, being less expensive than traditional methods, is strongly desired. One of the possible routes towards this end was initiated already in the early days of quantum mechanics. In the twenties, Thomas [5] and Fermi [6] developed the formalism, well rooted in quantum mechanics, from which the orbitals were completely eliminated. Orbital-free formalisms applicable to chemical systems would revolutionize computer modelling in chem- istry. Unfortunately, the Thomas-Fermi theory, although applicable to model physical systems, was shown to be useless in describing molecular systems. In 1962, Teller proved that this theory predicts that molecules are always unstable [7]. Despite this discouraging result many theoreticians picked up the Thomas-Fermi ideas. These works led to practical computational methods, in which the orbitals are partially or even completely eliminated from the formalism. The Kohn-Sham formalism [8], widely used in modelling, can be seen as one of such approaches. The present review deals with other approaches where the role of orbitals is reduced even further. It is organized in the following way. Firstly, we outline the role of the concept of orbitals in well-established theoretical formalisms currently in use. In the following part, we review the modern applications of the ideas by Thomas and Fermi leading to formalisms where the use of orbitals is completely eliminated, and present applications in computational studies relevant to materials science. In the subsequent parts we review approaches in which orbitals are eliminated partially. In these types of approaches, some terms of quantum mechanical origin in the total energy expression are approximated by means of orbital-free expressions. The long-standing interest of the author of this review lies in the development and applications of a formalism belonging to this group. The formulation of density functional theory, which uses or- 

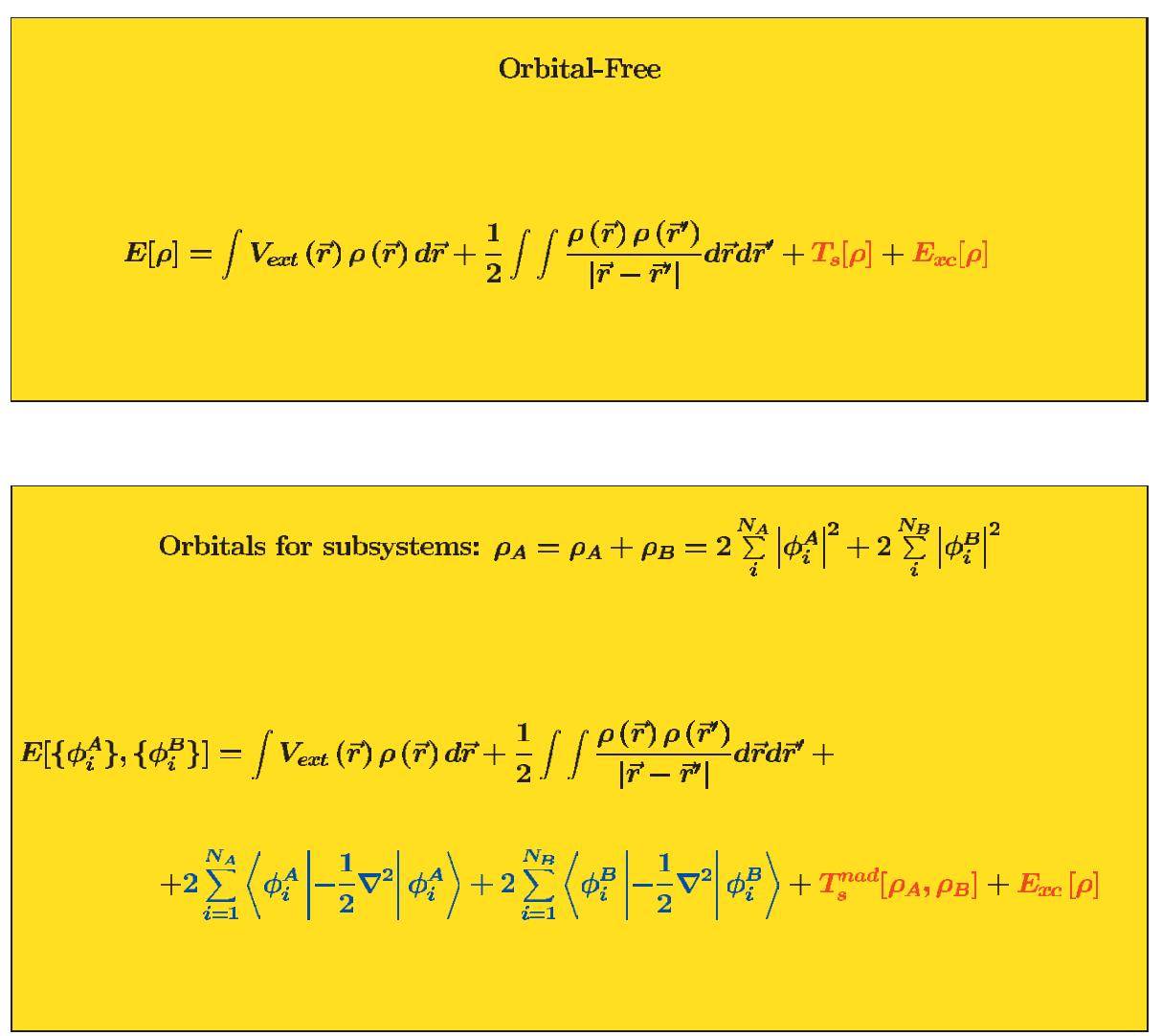

$$
\begin{gathered}
\text { Orbitals for the entire system (Kohn-Sham): } \rho=2 \sum_{i}^{N}\left|\phi_{i}\right|^{2} \\
E\left[\left\{\phi_{i}\right\}\right]=\int V_{\epsilon x t}(\vec{r}) \rho(\vec{r}) d \vec{r}+\frac{1}{2} \iint \frac{\rho(\vec{r}) \rho\left(\vec{r}^{\prime}\right)}{\left|\vec{r}-\vec{r}^{\prime}\right|} d \vec{r} d \vec{r}^{\prime}+2 \sum_{i=1}^{N}\left\langle\phi_{i}\left|-\frac{1}{2} \nabla^{2}\right| \phi_{i}\right\rangle+E_{x c}[\rho]
\end{gathered}
$$

Fig. The role of orbitals in three types of formalisms in density functional theory. The terms depending on orbitals are marked in blue. Blue and black colors are used for the terms of known exact analytic form. The terms, which are evaluated using approximate orbital-free expressions, are marked in red.

bitals localized in a pre-defined subsystem, and which has been developed and applied in our Geneva group, will be covered. The formulas for the total energy functionals discussed in this review are given in the Fig., which shall be used as a graphical guideline throughout the text.

\section{Orbitals in Chemistry}

Quantum mechanics brought to chemistry the concept of orbitals (atomic or molecular), which proved to be of inestimable value. The publication of the seminal work by Heitler and London in 1927 [9], which established the link between the key object in quantum chemistry, i.e. the wavefunction approximated by means of molecular orbitals and the fundamental object of interest in where the one-electron functions $\phi_{i}$ are called molecular orbitals. $\Psi\left(\vec{r}_{1}, \vec{r}_{2}, \ldots, \vec{r}_{N}\right)$ given in Eqn. (1) satisfies automatically the Pauli exclusion principle thus reflecting one of the key effects of a purely quantum mechanical origin. Except for one-electron systems, $\Psi\left(\vec{r}_{1}, \vec{r}_{2}, \ldots, \vec{r}_{N}\right)$ is only an approximation to the exact wavefunction due to its single-determinant form. In a general case, the exact wavefunction can be constructed as a sum of several functions of the $\Psi\left(\vec{r}_{1}, \vec{r}_{2}, \ldots, \vec{r}_{N}\right)$ form [10]. Nevertheless, even for systems comprising many electrons, the single-determinant approximation to the wavefunction proves to be extremely useful. It provides: the basis for the Aufbau principle, the qualitative description of the formation of covalent, ionic, and coordination bonds, chemical reactivity of functional groups, electron spectroscopy, magnetic properties, etc. Its conceptual usefulness is matched by various quantitative computational methods including that of Hartree and Fock and the semiempirical ones. We should mention, however, that the single-determinant approximation to the wavefunction (Eqn. (1)) proves inadequate to describe some effects even qualitatively (van der Waals interactions, dissociation of chemical bonds, etc.).

Density functional theory in its KohnSham formulation [8] provides another general theoretical framework in which orbitals are applied. It is important to underline the conceptual difference between two different uses of orbitals: $i$ ) in conventional wavefunction based approaches they are used to approximate the wavefunction as it is done in Eqn. (1) and ii) in the Kohn-Sham formalism where, formally speaking, they do not have any physical (chemical) meaning and are merely auxiliary quantities used to represent the ground-state electron density $(\rho)$ :

$$
\rho=2 \sum_{i}^{N} \phi_{i}^{*}(\vec{r}) \phi_{i}(\vec{r})
$$

The search for a physical interpretation of Kohn-Sham orbitals remains an appealing objective and much progress has been made in this direction marked by Janak's [11] theorem, which gave the physical meaning of the eigenvalue of the highest occupied KohnSham orbital as the negative of the ionization potential and the series of works from the Amsterdam group leading to the generalization of Koopman's theorem [12].

The indispensability of orbitals seems, therefore, overwhelmingly justified by experience accumulated over the years in the text):

$$
\Psi\left(\vec{r}_{1}, \vec{r}_{2}, \ldots, \vec{r}_{N}\right) \approx \Psi_{s}\left(\vec{r}_{1}, \vec{r}_{2}, \ldots, \vec{r}_{N}\right)=\frac{1}{\sqrt{N !}}\left|\begin{array}{cccc}
\phi_{1}\left(\vec{r}_{1}\right) & \phi_{1}\left(r_{2}\right) & \ldots \ldots & \phi_{1}\left(\vec{r}_{N}\right) \\
\phi_{2}\left(\vec{r}_{1}\right) & \phi_{2}\left(\vec{r}_{2}\right) & \ldots & \phi_{2}\left(\vec{r}_{N}\right) \\
\ldots . & \ldots . & \ldots & \ldots . \\
\phi_{N}\left(\vec{r}_{1}\right) & \phi_{N}\left(\vec{r}_{2}\right) & \ldots & \phi_{N}\left(\vec{r}_{N}\right)
\end{array}\right|
$$


computational chemistry. In almost all traditional quantum chemistry methods the total energy is explicitly expressed by means of orbitals. Nevertheless, attempts were made to eliminate orbitals from the expressions for the total energy. In a series of works started in 1951, Slater showed that the exchange energy could be reasonably approximated without using orbitals for molecular systems [13]. This brings us to the key issue of this review addressed in the next section.

\section{Are Orbitals Indispensable in De- scribing Molecules and Materials?}

Hohenberg and Kohn formulated the underlying theorems of density functional theory, which provide a negative answer to the above question [14]. This stands in obvious contradiction to generally held beliefs (e.g. [2]). According to the Hohenberg and Kohn theorems, the electron density for a given chemical system uniquely determines the ground-state energy. The total energy can thus be seen as a functional of the electron density $\mathrm{E}[\rho]$. In advanced calculus, a functional is a correspondence, which assigns a defined number ( $\mathrm{E}$ in our case) to each function belonging to some class ( $\rho$ in our case) [15]. Moreover, the minimum of $E[\rho]$ is just the ground-state energy. Many exact mathematical properties of this functional are known except for its analytic form. The direct application of the Hohenberg-Kohn theorems must rely on approximate expressions for $E[\rho]$. The headline question of this section might be, therefore, reformulated: "Are the orbital-free approximations to the total energy functional defined in the Hohenberg-Kohn theorems of any practical use in chemistry?"

Despite the original failure of the Thomas-Fermi approximation to the total energy functional, the search for its better approximation has been an object of continuous interest for theoreticians. Various theoretical routes have been explored in such studies leading to a number of much better approximations than the ones used originally by Thomas and Fermi. As a result, orbital-free computer modelling studies at a quantum mechanical level became reality. For instance, orbital-free methods have been applied in the studies concerning: structure and phase transitions in alkali metal clusters of the size up to one hundred atoms [16], dynamic properties of liquid $\mathrm{Li}$ and $\mathrm{Al}$ and liquid binary systems such as $\mathrm{LiNa}, \mathrm{LiMg}$, $\mathrm{LiBa}, \mathrm{NaCs}$, and $\mathrm{KC}$ [17], diffusion of defects in metals [18], surfaces [19], metal-insulator transition in quantum dots [20], structure and dynamics of solid-liquid interface in aluminum [21], pure $\mathrm{Al}$ or $\mathrm{Mg}$, and $\mathrm{Al}-\mathrm{Mg}$ alloys in crystalline phases [22]. Recently, Madden and coworkers showed that orbital- free strategy leads to the electronic structure of liquid metals almost indistinguishable from that derived from conventional orbitalbased calculations [23]. These examples concern complex materials. Unfortunately, not much can be found in the literature concerning applications of orbital-free methods to molecules. A notable exception is the recent work of Chan and Handy dealing with molecular systems [24], but the results are not very encouraging. Although the use of their orbital-free approximations to the total energy functional led to stable molecules, the equilibrium geometries were qualitatively wrong. In their present state, therefore, the orbital-free methods are no match to conventional orbital-based methods of quantum chemistry as far as the properties of small molecular systems are concerned. For a more comprehensive review of orbital-free methods and their applications, see [25]. For more recent developments, see the final report of the relevant workshop Computer modelling of atoms, molecules, and materials using approximate functionals of the kinetic energy we organized at CECAM in Lyon in summer 2002 [26].

\section{Orbitals in Kohn-Sham Formalism}

In this section, we turn back to the KohnSham formulation of density functional theory [8], which we see as a successful step towards the elimination of orbitals. In this formalism, several terms in the total energy of quantum mechanical origin are replaced by explicit functionals of the electron density. Nevertheless, the orbitals are its integral part (Fig.). At the minimum of total energy, the kinetic energy of the reference system of noninteracting electrons called conventionally $\mathrm{T}_{\mathrm{s}}[\rho]$, which is one of the components of the total energy, is expressed using explicitly the corresponding Kohn-Sham orbitals $\phi_{i}^{K S}$ (Fig.):

$T_{s}[\rho]=2 \sum_{i}^{N} \int \phi_{i}^{K S^{*}}(\vec{r})\left(-\frac{1}{2} \nabla^{2}\right) \phi_{i}^{K S}(\vec{r}) d \vec{r}$

where $\phi^{K S}$ indicates that the above equation holds for the orbitals derived from KohnSham equations.

All other components of the total energy functional $\mathrm{E}[\rho]$ defined in the HohenbergKohn theorems, i.e. the energy of the electron-electron repulsion, the energy of the electron-nuclear attraction, as well as the exchange-correlation energy are explicit functionals of electron density. Among these orbital-free terms, only the analytic form of the exchange-correlation functional is not known (Fig.). Experience shows that the orbital-free approximations to $\mathrm{E}_{\mathrm{xc}}[\rho]$ prove extremely useful in chemistry. The simplest one, called Local Density Approximation (LDA), in which the exchange-correlation functional is expressed by means of the electron density, leads to a very good structure (especially lengths of covalent bonds and bond angles), good vibrational properties, and electric moments, e.g. [27]. In the group of more sophisticated approximations called collectively Generalized Gradient Approximation (GGA), the exchange-correlation functional depends not only on the electron density but also on its gradient. GGA is usually a better approximation for practical purposes than LDA especially as far as the thermochemistry is concerned [28][29]. The general applicability of LDA and especially GGA in modelling chemical systems allows us to conclude that these approximations applied within the Kohn-Sham formalism represent successful milestones on the road towards elimination of orbitals. In these methods the orbitals are used only in one component of the total energy (Fig.). Unfortunately, no further milestones are currently in view on this road. The emergence of hybrid approximations such as the widely used B3LYP functional [30] shows that the re-introduction of orbitals in order to evaluate exchange-correlation energy is probably the direction to follow. The review article by Gill which appeared under a provocative title 'Obituary: Density Functional Theory (1927-1993)' provides a good illustration for such developments [31].

\section{Replacing the Kohn-Sham Orbitals for the Whole System by Localized Orbitals in the Total-energy Bi-func- tional Based Approach}

In 1972, Gordon and Kim proposed a model [32] in which the total energy of a complex formed by a pair of noble gas atoms was expressed as in the Thomas-Fermi theory. In their model the interaction energy is evaluated according to the formula:

$$
\mathrm{E}_{\text {int }}=\mathrm{E}\left[\rho^{0}{ }_{\mathrm{A}}+\rho^{0}{ }_{\mathrm{B}}\right]-\mathrm{E}\left[\rho^{0}{ }_{\mathrm{A}}\right]-\mathrm{E}\left[\rho^{0}{ }_{\mathrm{B}}\right]
$$

where the functional $E[\rho]$ is expressed as in orbital-free methods (Fig.), and the $\rho^{0}{ }_{\mathrm{A}}$ and $\rho^{0}{ }_{B}$ are the electron densities of the isolated atoms $\left(\rho^{0}{ }_{A}+\rho^{0}{ }_{B}\right.$ is thus a superposition of the electron densities of the two isolated atoms).

Astonishingly, even simple approximations to all relevant functionals applied in Eqn. (4) led to good interaction energies for some systems. For $\mathrm{Ne}-\mathrm{Ne}, \mathrm{Ar}-\mathrm{Ar}$, and $\mathrm{Kr}-\mathrm{Kr}$ dimers, they agree with experimental values within less than $15 \%$. The potential energy curves show, however, a tendency towards too short equilibrium distances, whereas their asymptotic behavior at large distances is qualitatively wrong (exponential instead of proportional to $-1 / \mathrm{R}^{6}$ ). Evaluation of the interaction energy using Eqn. (4) requires, however, a prior knowledge of $\rho^{0}{ }_{\mathrm{A}}$ and $\rho^{0}{ }_{\mathrm{B}}$ because the Gordon-Kim model is not based 
on a variational principle. It is worthwhile to notice that the right-hand side of Eqn. (4) defines a bi-functional, i.e. a functional depending on two electron densities $\left(\rho^{0}{ }_{A}\right.$ and $\left.\rho^{0}{ }_{\mathrm{B}}\right)$. For a system comprising more than two components, one can define a multi-functional which depends on electron densities of several subsystems. In 1991, Cortona formulated a general formalism in which the totalenergy multifunctional was used in variational calculations following the second Hohenberg-Kohn theorem [33]. As a result, both the total energy and the electron densities of all subsystems can be obtained from the EulerLagrange type of minimization.

In the case of two interacting subsystems (intermolecular complexes for instance), partitioning the total electron density $(\rho)$ into two components $\rho_{\mathrm{A}}$ and $\rho_{\mathrm{B}}$, each comprising an integer number of electrons such that $\rho=$ $\rho_{A}+\rho_{B}$, allows one to express the total kinet-

$$
\mathrm{T}_{\mathrm{s}}\left[\rho_{\mathrm{A}}+\rho_{\mathrm{B}}\right]=\mathrm{T}_{\mathrm{s}}\left[\rho_{\mathrm{A}}\right]+\mathrm{T}_{\mathrm{s}}\left[\rho_{\mathrm{B}}\right]+\mathrm{T}_{\mathrm{s}}{ }^{\mathrm{nad}}\left[\rho_{\mathrm{A}}, \rho_{\mathrm{B}}\right]
$$

ic energy $\left(T_{S}\left[\rho_{A}+\rho_{B}\right]\right)$ in a hybrid way [34]:

At the energy minimum, the terms $\mathrm{T}_{\mathrm{S}}\left[\rho_{\mathrm{A}}\right]$ and $\mathrm{T}_{\mathrm{s}}\left[\rho_{\mathrm{B}}\right]$ can be calculated using KohnSham-like orbitals and Eqn. (3). The term $\mathrm{T}_{\mathrm{s}}{ }^{\mathrm{nad}}\left[\rho_{\mathrm{A}}, \rho_{\mathrm{B}}\right]$ can be evaluated as an orbitalfree bi-functional for any pair of densities.

As a consequence, also the total energy can be represented as a functional of twoelectron densities $\left(E\left[\rho_{A}, \rho_{B}\right]\right)$ or as a functional of two sets of orbitals in practical calculations (Fig.). It is worthwhile to notice that the third component on the right-hand-side of Eqn. (5), i.e. $\mathrm{T}_{\mathrm{S}}{ }^{\mathrm{nad}}\left[\rho_{\mathrm{A}}, \rho_{\mathrm{B}}\right]$ is expected to be small. (In the case of non-overlapping $\rho_{A}$ and $\rho_{B}$ it disappears [35].) Approximating it and applying it in practical modelling can be expected, therefore, to face less formidable difficulties than the ones encountered by the orbital-free formalisms, where the entire kinetic energy must be evaluated using an approximate functional.

Variational use of the bi-functional $\mathrm{E}\left[\rho_{\mathrm{A}}, \rho_{\mathrm{B}}\right]$ can be seen, therefore, as the intermediate strategy to develop practical computational methods which lie between the Kohn-Sham one and orbital-free ones (Fig.). In this formalism, the need to use orbitals to construct the electron density of the whole system, which lies at the origin of the difficulties in applying Kohn-Sham formalism to very large systems, is partially eliminated owing to partitioning the total kinetic energy $(\mathrm{T}[\rho])$.

Cortona applied the multifunctional $\mathrm{E}\left[\rho_{1}, \rho_{2}, . ., \rho_{\mathrm{N}}\right]$ to study solids. Despite rough simplifications made in the computer implementation such as assuming that each $\rho_{\mathrm{i}}$, representing the electron density of an atom in a crystal is spherically symmetric, and using rather simple approximations for the relevant functionals, the structure and elastic properties of the crystals studied were in a very good agreement with experimental data. The computer implementation of this formalism for studies of solids was recently improved and applied to derive various properties of solids such as $\mathrm{NaCl}, \mathrm{MgO}$, and AlP [36].

The first application of the bi-functional $\mathrm{E}\left[\rho_{\mathrm{A}}, \rho_{\mathrm{B}}\right]$ in variational calculations for molecules was made in the context of orbital-free embedding by Wesolowski and Warshel in 1993 [34]. In 1995, we developed a universal computer implementation of this formalism allowing us to use the bi-functional in $E\left[\rho_{A}, \rho_{B}\right]$ in variational calculations for molecular systems [35]. This allowed us to conduct systematic studies aimed at development of good approximations to the relevant bi-functionals $\mathrm{T}_{\mathrm{S}}{ }^{\mathrm{nad}}\left[\rho_{\mathrm{A}}, \rho_{\mathrm{B}}\right]$ and $\mathrm{E}\left[\rho_{\mathrm{A}}, \rho_{\mathrm{B}}\right]$. We followed three strategies: a) numerical comparisons with the exact reference data for $\mathrm{T}_{\mathrm{s}}{ }^{\mathrm{nad}}\left[\rho_{\mathrm{A}}, \rho_{\mathrm{B}}\right][38-40]$, b) mathematical considerations involving exact properties of $\mathrm{T}_{\mathrm{S}}{ }^{\mathrm{nad}}\left[\rho_{\mathrm{A}}, \rho_{\mathrm{B}}\right]$ [37], and c) analysis of the

overall accuracy of interaction energies derived using a given approximation to $E\left[\rho_{A}, \rho_{B}\right]$ [41]. From these studies, it can be concluded that the gradient-dependent approximation to $\mathrm{T}_{\mathrm{S}}{ }^{\mathrm{nad}}\left[\rho_{\mathrm{A}}, \rho_{\mathrm{B}}\right]$ proposed in 1996 [39][40], which uses the approximate functional of the kinetic energy developed by Lembarki and Chermette [42], is the best one for systems where the $\rho_{A}, \rho_{B}$ overlap is small. Moreover, using it together with the PW91 [43] approximation to the exchange-correlation functional results in a very good approximation to the bi-functional of the total energy $E\left[\rho_{A}, \rho_{B}\right]$ for such pairs of electron densities as the ones occurring in van der Waals complexes close to the equilibrium geometry [41]. These methodological and practical developments allowed us to study various intermolecular systems by performing fully variational calculations (minimizing $E\left[\rho_{A}, \rho_{B}\right]$ by varying both $\rho_{A}$ and $\rho_{B}$ ) or constrained minimization of $E\left[\rho_{A}, \rho_{B}\right]$ by varying only $\rho_{A}$ with a frozen $\rho_{B}$. The fully variational calculations were applied in studies of such systems as: complexes of small diatomics with benzene [46] or carbazole [47], the benzene dimer [44], and complexes involving $\mathrm{H}_{2}$ and various polycyclic aromatic hydrocarbons [45]. The second type of calculations allows one to derive the electronic structure of an embedded molecule from Kohn-Sham-like one-electron equations. Here, the effect of the interactions between and the embedded molecule and its surroundings is expressed exactly by means of an orbital-free embedding potential [34]:

where:

$N_{B}$ is the number of the nuclei in subsystem $\mathrm{B} ; \mathrm{Z}_{\alpha}{ }^{\mathrm{B}}$ is the nuclear charge of nucleus $\alpha$ in subsystem $B$; the exchange-correlation functional $\left(\mathrm{E}_{\mathrm{xc}}[\rho]\right)$ is defined as in the Kohn-Sham formalism [8].

It is worthwhile to notice that the embedding effective potential of Eqn. (6) does not depend on orbitals in the subsystems B but only on its electron density $\left(\rho_{\mathrm{B}}\right)$; it is, therefore, orbital-free. Such a formalism is suited for cases where the primary interest lies in an embedded molecule whereas its environment can be satisfactorily described using a simpler model. Several authors applied the orbital-free embedding potential of Eqn. (5) in various contexts [48-50].

The area of applicability of the practical formalism based on the total-energy bifunctional and using the relevant approximations covers all such chemical systems, which can be partitioned into two subsystems held together by non-bonding interactions (electrostatic and van der Waals forces). We have applied it to: a) derive the hydration energy differences using combined QM/MD simulations and to analyze fluctuations of the dipole moment of an individual water molecule in liquid phase [51], b) determine the solvent effect on the proton transfer in a hydrated model system [52], c) determine the hyperfine structure of a model radical system in noble gas matrices [53], d) determine the vibrational properties of molecules physisorbed on metal oxide surfaces [54] and in zeolites [55], and e) analyze the potential energy curve corresponding to the proton transfer in an enzyme active center [56]. Currently, modelling studies applying the orbital-free embedding formalism for biological systems are carried out by Warshel and coworkers [57]. Such applications have already been reviewed in an article in this journal [58] and will not be discussed in more detail here. Recently, we combined the orbital-free embedding formalism with the linear-response density functional theory to study excited states of embedded molecules [59].

\section{Concluding Remarks}

In this review, we outlined recent developments in pursuing the long-standing and challenging objective to develop a universal computational approach based on quan-

$$
\begin{aligned}
V^{e m b} & =V^{e m b(K S C E D)}\left[\vec{r}, \rho_{A}, \rho_{B}\right]= \\
& =-\sum_{\alpha}^{N_{B}} \frac{Z_{\alpha}^{B}}{\left|\vec{R}_{\alpha}-\vec{r}\right|}+\int \frac{\rho_{B}\left(\vec{r}^{\prime}\right)}{\left|\vec{r}-\vec{r}^{\prime}\right|} d r^{\prime}+\left.\frac{\delta E_{x c}[\rho]}{\delta \rho}\right|_{\rho=\rho_{A}+\rho_{B}}-\left.\frac{\delta E_{x c}[\rho]}{\delta \rho}\right|_{\rho=\rho_{A}}+\frac{\delta T_{s}^{n a d}\left[\rho_{A}, \rho_{B}\right]}{\delta \rho_{A}}
\end{aligned}
$$


tum mechanics, which is applicable to chemical systems but does not use orbitals. This quest, although it did not achieve its original objective, brought many pay-offs in the form of less universal formalisms in which the orbitals are either completely or partially eliminated: a) orbital-free methods which are direct descendants of the ideas of Thomas and Fermi where no orbitals are used, b) methods based on partitioning of the kinetic energy, in which the exchangecorrelation and non-additive kinetic energy components of the total energy are expressed as orbital-free functionals, and c) methods based on the Kohn-Sham equations (LDA and GGA), in which orbitals are used only to calculate the kinetic energy component of the total energy, whereas all other components are evaluated as orbitalfree functionals.

The last among the three approaches, i.e. the formalism of Kohn and Sham, in which the orbitals are used to construct the total electron density and to evaluate the kinetic energy, is the most universal one. Although improvements of the approximations to the exchange-correlation functional are highly desired, it is currently used in almost all branches of chemistry. The two other approaches discussed here are less universally applicable.

Currently, the domain of applicability of methods in which the orbitals are completely eliminated is limited to such systems where the collective behavior of a large number of electrons and nuclei determines their overall properties. As far as details of molecular structure are concerned, such methods are still far inferior to even the simplest molecular-orbital-based methods.

Concerning kinetic-energy-partitionbased methods, we showed their general usefulness in various types of calculations. In particular, they allow one to obtain very accurate details of the electronic structure in a selected fragment of a complex material without constructing orbitals representing the entire investigated system (orbitalfree embedding). At the current stage, their domain of applicability is limited to such systems which can be partitioned into subsystems not linked by covalent bonds. We are also continuing working on further improvement of the relevant approximate functionals [60][61].

\section{Acknowledgements}

The reviewed works on the total energy bifunctional and the orbital-free embedding have been supported by the grants from FNRS (project 21-63645.00) and CSSC in Mano and by the Action D26 of COST.

Received: March 15, 2004

[1] R.F.W. Bader, Intl. J. Quant. Chem. 2003, 94, 173.

[2] G. Frenking, Angew. Chem. Int. Ed. 2003,
$42,143$.

[3] In a recent book, 'The New Chemistry - A showcase for modern chemistry and its applications' Ed. N. Hall, Cambridge University Press, Cambridge 2000; most of the chapters deal with systems comprising more than one hundred atoms.

[4] G.E. Scuseria, J. Phys. Chem. 1999, 103, 4782.

[5] E. Fermi, Z. Phys. 1928, 48, 73.

[6] L.H. Thomas, Proc. Camb. Phil. Soc. 1927, 23, 542.

[7] E. Teller, Rev. Mod. Phys. 1962, 34, 627.

[8] W. Kohn, L. J. Sham, Phys. Rev. 1965, 140, A1133.

[9] W. Heitler, F. London, Z. Phys. 1927, 44, 455.

[10] A. Szabo, N.S. Ostlund, 'Modern Quantum Chemistry', McGraw-Hill, Inc. 1989.

[11] J.F. Janak, Phys. Rev. 1978, 18, 7165.

[12] O. Gritsenko, E.-J. Baerends, J. Chem. Phys. 2002, 117, 9154.

[13] J.C. Slater, Phys. Rev. 1951, 81, 385.

[14] P. Hohenberg, W. Kohn, Phys. Rev. 1964 136, B864.

[15] I.M. Gelfand, S.V. Fomin, 'Calculus of variations', Dover Publications Inc. Mineola, New York, 1963, p 1.

[16] A. Aguado, J.M. Lopez, J.A. Alonso, M.J. Stott, J. Phys. Chem. B. 2001, 105, 2386; A. Aguado, L.M. Molina, J.M. Lopez, J.A. Alonso, Eur. Phys. J. D. 2001, 15, 221.

[17] J. Blanco, D.J. Gonzalez, L.E. Gonzalez, J.M. Lopez, M.J. Stott, J. Non-Crystalline Solids 2002, 312, 148.

[18] E. Smargiassi, Phys. Rev. B. 2001, 65, 012301.

[19] Y.A. Wang, N. Govind, E.A. Carter, Phys. Rev. B. 1999, 60, 16350.

[20] S.C. Watson, E.A. Carter, Comp. Phys. Commun. 2000, 128, 67.

[21] B.J. Jesson, P.A. Madden, J. Chem. Phys. 2000, 113, 5935

[22] K.M. Carling, E.A. Carter, Modelling and Simulation in Materials Science and Engineering 2003, 11. 339.

[23] J.A. Anta, P.A. Madden, Phys. Cond. Matt 1999, 11, 6099.

[24] G. Chan, N.C. Handy, J. Chem. Phys. 2001, 114, 631.

[25] Y.A. Wang and E.A. Carter, in 'Theoretical Methods in Condensed Phase Chemistry', Ed. Schwartz, p. 117-184, Kluwer, Dordrecht, 2000.

[26] CECAM workshop: 'Computer modelling of atoms, molecules, and materials using approximate functionals of the kinetic energy' at CECAM, July 31-August 2, 2002, Lyon (France) organized by T.A. Wesolowski and H. Chermette. The official report of the workshop is available at: http://lcta.unige.ch/ tomek/cecam2002/ cecam2002.html

[27] J. Andzelm, E. Wimmer, J. Chem. Phys 1992, 96, 1280

[28] A. Becke, J. Chem. Phys. 1992, 96, 2155.

[29] A. Becke, J. Chem. Phys. 1992, 97, 9173.

[30] A. Becke, J. Chem. Phys. 1993, 98, 5648

[31] P.W. Gill, Austral. J. Chem. 2002, 54, 661.

[32] R.G. Gordon, Y.S. Kim, J. Chem. Phys. 1972, 56, 3122.

[33] P. Cortona, Phys. Rev. B 1991, 44, 8454.

[34] T.A. Wesolowski, A. Warshel, J. Phys.
Chem. 1993, 97, 8050.

[35] T.A. Wesolowski J. Phys. A: Mathematical and General: 2003, 36, 10607.

[36] L.L. Boyer, H.T. Stokes, M.J. Mehl, Phys. Rev. Lett. 2000, 84, 709.

[37] T.A. Wesolowski, J. Weber, Chem. Phys. Lett. 1996, 248, 71.

[38] T.A. Wesolowski, J. Weber, Intl. J. Quant. Chem. 1997, 61, 303.

[39] T.A. Wesolowski, H. Chermette, J. Weber, J. Chem. Phys. 1996, 105, 9182.

[40] T.A. Wesolowski, J. Chem. Phys. 1997, 106,8516

[41] T.A. Wesolowski, F. Tran, J. Chem. Phys. 2003, 118, 2072

[42] A. Lembarki, H. Chermette, Phys. Rev. A. 1994, 50, 5328.

[43] J.P. Perdew, in 'Electronic Structure of Solids'91', Eds. P. Ziesche, H. Eschrig, Academie Verlag, Berlin 1991, p. 11.

[44] F. Tran, J. Weber, T.A. Wesolowski, Helv. Chim. Acta 2001, 84, 1489.

[45] F. Tran, J. Weber, T.A. Wesolowski, F. Cheikh, Y. Ellinger, F. Pauzat, J. Phys. Chem. B. 2002, 106, 8689.

[46] T.A. Wesolowski, Y. Ellinger, J. Weber, $J$. Chem. Phys. 1998, 108, 6078.

[47] T.A. Wesolowski, P.-Y. Morgantini, J. Weber, J. Chem. Phys. 2002, 116, 6411.

[48] E.V. Stefanovitch, T.N. Truong, J. Chem. Phys. 1996, 104, 2946.

[49] J. Trail, D. Bird, Phys. Rev. B 2000, 62, 16402.

[50] N. Govind, Y.A. Wag, A.J.R. da Silva, E.A. Carter, Chem. Phys. Lett. 1999, 295, 129.

[51] T.A. Wesolowski, A. Warshel, J. Phys. Chem. 1994, 98, 5183.

[52] T.A. Wesolowski, R.P. Muller, A. Warshel, J. Phys. Chem. 1995, 100, 15444.

[53] T.A. Wesolowski, Chem. Phys. Lett. 1999, $311,87$.

[54] N. Vulliermet, T.A. Wesolowski, J. Weber, Collect. Czech. Chem. Commun. 1998, 63, 1447.

[55] T.A. Wesolowski, A. Goursot, J. Weber, $J$. Chem. Phys. 2001, 115, 4791.

[56] G. Hong, M. Strajbl, T. A. Wesolowski, A. Warshel, J. Comput. Chem. 2000, 21, 1554.

[57] M.H.M. Olsson, G.Y. Hong, A. Warshel, J. Am. Chem. Soc. 2004, 96, 577.

[58] T. Wesolowski, Chimia 2002, 56, 707.

[59] M. Casida, T. Wesolowski, Intl. J. Quant. Chem. 2004, 96, 577.

[60] F. Tran, T.A. Wesolowski, Int. J. Quant. Chem. 2002, 89, 441.

[61] F. Tran, T.A. Wesolowski, Chem. Phys. Lett. 2002, 360, 209. 